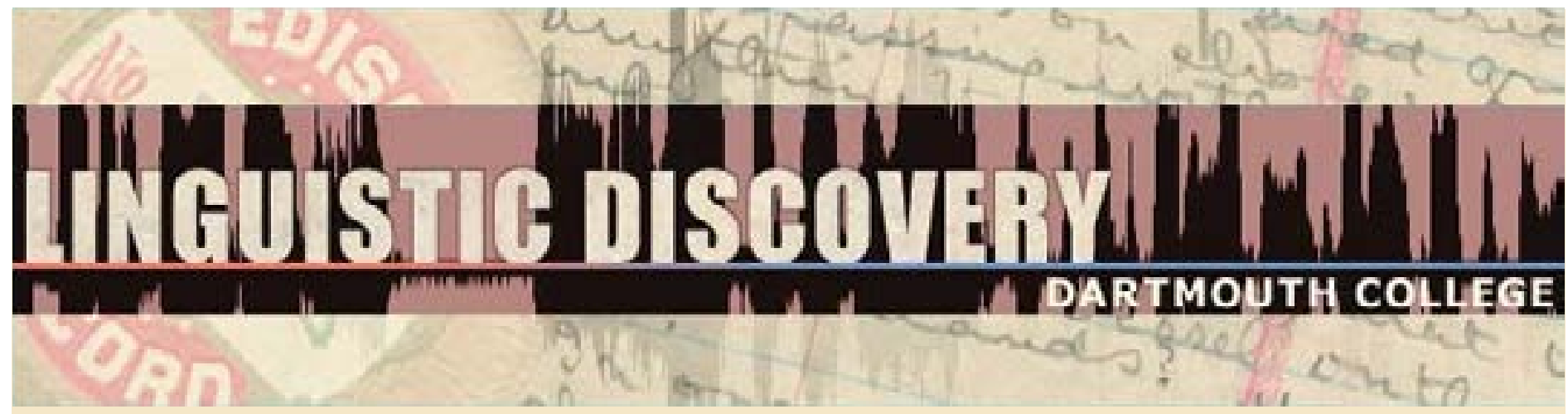

\begin{tabular}{|l|}
\hline Volume 4 \\
Issue 1 \\
2006 \\
\hline
\end{tabular}

\title{
A Cross-linguistic Corpus of Forms Meaning "Yes"
}

Steve Parker

SIL International and Graduate Institute of Applied Linguistics

doi: $10.1349 / P S 1.1537-0852 . A .306$

url: http://journals.dartmouth.edu/cgi-bin/WebObjects/ Journals.woa/1/xmlpage/1/article/306

\section{Linguistic Discovery}

Published by the Dartmouth College Library Copyright to this article is held by the authors. ISSN 1537-0852 linguistic-discovery.dartmouth.edu 


\title{
A Cross-linguistic Corpus of Forms Meaning 'yes'
}

\author{
Steve Parker \\ SIL International and Graduate Institute of Applied Linguistics
}

Based on a carefully-compiled database of 604 attested forms for 'yes' taken from 512 languages spoken in over 70 countries, I show that this word exhibits a cross-linguistic tendency to contain laryngeal phonemes $(/ \mathrm{R} /$ or $/ \mathrm{h} /)$. As part of the statistical analysis I examine cognate items within specific genetic families and argue that certain phonotactic patterns involving 'yes' are not random in nature. These findings further corroborate the observation that glottal consonants often behave phonologically as a default or unmarked class of segments.

\section{Introduction}

A very basic and important aspect of natural human language is the fact that in the great majority of cases, the relationship between a word's meaning and its pronunciation is arbitrary and unpredictable. Exceptions to this generalization of non-iconicity are therefore noteworthy. The purpose of this paper is to document a striking pattern I have discovered in languages from all areas of the world. Specifically, the lexical item meaning 'yes' has a fairly strong tendency to contain one or more glottal consonants - either [h] and/or [?]. In the next section I present a corpus of forms listing the word(s) for 'yes' in 512 languages belonging to 64 major linguistic families and show that this phenomenon (laryngeal consonantism) is attested in at least 604 specific occurrences. In the ensuing discussion I give summary statistics and conclude that several common phonological themes occur with a frequency that is almost certainly greater than chance. The presentation here builds on previous work in Parker $(1996,2006)$. In the former paper I introduced the main pattern but was only able to include a truncated corpus of 44 forms due to limitations on space. And in the latter article (in Spanish) the organization of the word list by country obscures certain typological facts that are more directly elucidated here. The present paper constitutes the first full analysis in English of the entire corpus of 604 words, arranged and discussed according to genetic affiliation.

\section{Data}

In Table 1 below I list a series of lexical items meaning 'yes' in 512 specific languages. As noted above, the criterion for including a word in this corpus is any form for 'yes' which contains one or more instances of either or both glottal consonants - [h] and/or [?], since this is the common pattern I have identified and propose to analyze here. I transcribe the items using IPA characters and generally repeat as much phonetic detail as each source reports. In a small number of cases it is not clear which (non-laryngeal) sound is being represented, so I simply reproduce the original symbols here, e.g., $\ddot{a}$. Some of my sources transcribe the items phonetically, indicating complete surface realizations, while other sources use a more abstract, phonemic level of representation. However, since it is not always clear which of these two options is intended, I just faithfully copy each word below without indicating any distinction between different levels of phonological analysis. Nevertheless, there is one significant exception to this procedure which I consistently follow: in my data below I do not include any instances of word-initial [?]'s when it is clear from the source that these are not phonemic. This is due to the very common crosslinguistic tendency for languages to epenthesize a phonetic [?] as an automatic reflex to fulfill the 
requirement for a syllable onset in phrase-initial or word-initial position. Consequently, since this nearly universal process would obviously confound my results here by greatly (and artificially) increasing the sample size, I exclude all such example words from my data. I include forms with a word-initial glottal stop only when it is clear from the source that that segment is contrastive in that position in that language. Therefore, all cases of initial [?]'s in Table 1 below are assumed to be phonemic, as far as I am aware.

The data items I present here come from many different types of sources, and span over a decade of compilation. Long ago it became unwieldy to keep track of each reference, so I cannot list all of them in my bibliography section. Nevertheless, in all cases my preference is to rely on primary sources whenever possible. Consequently, the majority of these forms have been taken from published reference works such as dictionaries, descriptive grammars, etc. When feasible I also try to communicate directly in person with a linguist who has done extensive fieldwork on the language in question, or with a native speaker. However, for a relatively small number of cases I am not aware of a published source since at times I have included some data from places such as the Internet, survey reports by my SIL colleagues, etc. Consequently, it is not unlikely that a few transcriptional errors may have crept into my corpus. Nevertheless, given the overall robustness of the patterns I have observed in data from sources that are more reliable, none of my general conclusions are in doubt, as I will discuss in the next section.

Another issue which merits comment is the meaning of the items displayed in my list in Table 1 below. To the best of my knowledge, all of the words I present here are citation forms for 'yes' which are considered the standard, official way to express the concept of verbal assent, as in response to a yes/no question, for example. Many languages also have less formal equivalents, such as the English affirmation grunt typically written $u h$-uh (or in similar fashion). This type of expression is in fact very common, perhaps almost universal, so I have tried to filter it out of my corpus so as not to inappropriately inflate the statistics. Consequently, in the compilation of my word list I have purposely excluded all forms translated as 'yes' but which are specifically noted to be slang, informal, non-standard, etc. A related detail is that some languages do not have a single word exactly equivalent to 'yes,' but instead use a phrase meaning something like 'it is good.' In a very few cases of this type I have included such forms in my corpus, but always and only with the condition that the language in question must not have any other simpler and more direct way to express assent, and thus a published work such as a dictionary has listed this expression as the closest equivalent for 'yes.'

Before presenting the actual data, I should clarify that no attempt has been made to balance the sample of languages included here, either in terms of their linguistic affiliation or their areal locations, unlike the ideal list put together for typological purposes in WALS (Haspelmath et al. 2005; cf. Whaley 1997). Rather, Table 1 below includes every form for 'yes' I have discovered to date which meets the criterion spelled out above (a glottal consonant). As such, certain genetic families are represented very heavily, while certain others are not represented at all. Likewise, some continents have many languages with matching forms, while others have relatively few. This fact will make it difficult to extrapolate inferential statistics about the word 'yes' for the planet as a whole, but that is not my primary concern here. Rather, in offering this corpus I simply wish to document all the words for 'yes' with a laryngeal consonant that I am aware of, for the sake of exhaustivity. Consequently, there are hundreds of languages whose forms I have purposely excluded from this list, such as English yes and Spanish sí. In fact, the total number of languages I surveyed for this study was about 1372, of which 512 have one or more matching forms, so the overall hit rate for my sample is about 37\%. After I give the attested forms I will return to these points and discuss them more systematically. 
I now describe the internal structure of my corpus, as displayed in Table 1 below. For the spelling of language names and countries I follow the latest edition of the Ethnologue (Gordon 2005). I also follow this reference for the linguistic affiliation (genetic classification) of all the languages. (Ethnologue itself bases its organization of linguistic relationships on Frawley 2003.) The order of presentation of the languages in Table 1 is by family, following the geographical scheme of WALS, which in turn is derived from that of Ruhlen (1987). Within each first-order macro-group (phylum) or stock, the subfamilies are arranged alphabetically, again following $W A L S$. Normally each family is broken down as far as the level of the genera posited by $W A L S$, with a few minor deviations motivated by Ethnologue. After the name of each family, subfamily, and genus, I note in parentheses the number of languages from that group which appear in my corpus. Within each mini-table I list three pieces of information, from left to right: (1) the name of the language, (2) the official name of the country or countries where it is (or was) mainly spoken, and (3) the word or words meaning 'yes,' separated by commas. In cases when a language is spoken in more than one country, the one I list first is considered primary by Ethnologue. The order of the languages in the leftmost column of each mini-table is alphabetical.

Table 1: Corpus of forms meaning 'yes' (or 'affirmation')

Niger-Congo (20 languages), Atlantic-Congo (19 languages), Atlantic (1 language)

\begin{tabular}{|l|l|l|}
\hline language & country & word(s) for 'yes' \\
\hline \hline Jola-Fonyi & Senegal & ahej, ehe \\
\hline
\end{tabular}

Niger-Congo (20), Atlantic-Congo (19), Volta-Congo (18), Benue-Congo (10), Bantoid (9)

\begin{tabular}{|l|l|l|}
\hline Akoose & Cameroon & Ree, Pẽẽ \\
\hline Digo & Kenya, Tanzania & èđé \\
\hline Fang & Equatorial Guinea & èhè \\
\hline Gikuyu & Kenya & eeh \\
\hline Kwanyama & Angola, Namibia & heeno \\
\hline Langi & Tanzania & Pèhé: \\
\hline Mbala & Democratic Republic of the Congo & êe \\
\hline Shona & Zimbabwe & ehe \\
\hline Venda & South Africa, Zimbabwe & ih \\
\hline
\end{tabular}

Niger-Congo (20), Atlantic-Congo (19), Volta-Congo (18), Benue-Congo (10), Nupoid (1)

\begin{tabular}{|l|l|l|}
\hline Nupe-Nupe-Tako & Nigeria & hin(jí) \\
\hline
\end{tabular}

Niger-Congo (20), Atlantic-Congo (19), Volta-Congo (18), Kru (1)

\begin{tabular}{|l|l|l|}
\hline (Abu) Dida & Côte d'Ivoire & hẽ $\tilde{\varepsilon}$ \\
\hline
\end{tabular}


Niger-Congo (20), Atlantic-Congo (19), Volta-Congo (18), Kwa (3)

\begin{tabular}{|c|c|c|}
\hline Akan & Ghana & $\varepsilon h \tilde{e} \tilde{e}$ \\
\hline $\mathrm{Ga}$ & Ghana & $\mathrm{h} \tilde{\varepsilon}$ \\
\hline Gen & Togo & heinn \\
\hline
\end{tabular}

Niger-Congo (20), Atlantic-Congo (19), Volta-Congo (18), North (4), Adamawa-Ubangi (2)

\begin{tabular}{|l|l|l|}
\hline Mbum & Cameroon, Central African Republic & ó?ó \\
\hline Zande & Democratic Republic of the Congo, Central African Republic & hein \\
\hline
\end{tabular}

Niger-Congo (20), Atlantic-Congo (19), Volta-Congo (18), North (4), Gur (2)

\begin{tabular}{|l|l|l|}
\hline Konni & Ghana & wă? \\
\hline Wali & Ghana & e?e \\
\hline
\end{tabular}

Niger-Congo (20), Mande (1), Western (1)

\begin{tabular}{|l|l|l|}
\hline Mandinka & Senegal & haa \\
\hline
\end{tabular}

Afro-Asiatic (10), Berber (2)

\begin{tabular}{|l|l|l|}
\hline Kabyle & Algeria & ih \\
\hline Tachelhit & Morocco & ihe \\
\hline
\end{tabular}

Afro-Asiatic (10), Chadic (1), West (1)

\begin{tabular}{|l|l|l|}
\hline Hausa & Nigeria & toh \\
\hline
\end{tabular}

Afro-Asiatic (10), Cushitic (2)

\begin{tabular}{|l|l|l|}
\hline Kambaata & Ethiopia & ?ãã \\
\hline Somali & Somalia & haa \\
\hline
\end{tabular}

Afro-Asiatic (10), Semitic (5)

\begin{tabular}{|l|l|l|}
\hline Assyrian Neo-Aramaic & Iraq & he \\
\hline Iraqi Arabic & Iraq & ?ii \\
\hline Moroccan Spoken Arabic & Morocco & ih \\
\hline Syrian (North Levantine Spoken) Arabic & Syria & ?ee \\
\hline Tigrigna & Ethiopia & ?uwej \\
\hline
\end{tabular}

Indo-European (23), Armenian (1)

\begin{tabular}{|l|l|l|}
\hline (Eastern) Armenian & Armenia & ha \\
\hline
\end{tabular} 
Indo-European (23), Celtic (1)

\section{\begin{tabular}{|l|l|l|}
\hline Scottish Gaelic & United Kingdom & haa \\
\hline
\end{tabular}}

Indo-European (23), Indo-Iranian (19), Indo-Aryan (15)

\begin{tabular}{|l|l|l|}
\hline Assamese & India, Bangladesh & haa \\
\hline Bengali & Bangladesh & ha \\
\hline Caribbean Hindustani & Suriname & han \\
\hline Eastern Panjabi & India & ha(n) ji \\
\hline Gujarati & India & haan \\
\hline Hindi & India & hai, haza, ha(an) \\
\hline Indus Kohistani & Pakistan & ah \\
\hline Kashmiri & India, Pakistan & ho \\
\hline Lambadi & India & hawə \\
\hline Marathi & India & ho \\
\hline Nepali & Nepal, India & haa \\
\hline Panjabi & Pakistan, India & hãã \\
\hline Romani & Romania & hai \\
\hline Sindhi & Pakistan, India & ha \\
\hline Urdu & Pakistan, India & hãã, ji hã, ha(3i) \\
\hline
\end{tabular}

Creole, Assamese based (Indo-European, Indo-Iranian, Indo-Aryan) (1)

\begin{tabular}{|l|l|l|}
\hline Naga Pidgin & India & hoi \\
\hline
\end{tabular}

Indo-European (23), Indo-Iranian (19), Iranian (3)

\begin{tabular}{|l|l|l|}
\hline Balochi & Pakistan, India & han \\
\hline Central Kurdish & Iraq & hari \\
\hline Pashto & Pakistan, Afghanistan & hoo \\
\hline
\end{tabular}

Indo-European (23), Slavic (2)

\begin{tabular}{|l|l|l|}
\hline Slovak & Slovakia & hej \\
\hline Upper Sorbian & Germany & haj \\
\hline
\end{tabular}

Uralic (1), Finnic (1)

\begin{tabular}{|l|l|l|}
\hline Estonian & Estonia & jah \\
\hline
\end{tabular}

Altaic (3), Turkic (3)

\begin{tabular}{|l|l|l|}
\hline Azerbaijani & Azerbaijan & hæ̃ \\
\hline Turkmen & Turkmenistan & hawa \\
\hline Uyghur & China & hä?ä \\
\hline
\end{tabular}


Japanese (1)

\begin{tabular}{|l|l|l|}
\hline Japanese & Japan & hai(?), eei \\
\hline
\end{tabular}

North Caucasian (2)

\begin{tabular}{|l|l|l|}
\hline Chechen & Russia & ha? \\
\hline Ingush & Russia & hwa?a \\
\hline
\end{tabular}

Dravidian (1), Southern (1)

\begin{tabular}{|l|l|l|}
\hline Kannada & India & haudu \\
\hline
\end{tabular}

Sino-Tibetan (10), Chinese (2)

\begin{tabular}{|l|l|l|}
\hline Hakka Chinese & China & hé \\
\hline Yue Chinese & China & hai \\
\hline
\end{tabular}

Sino-Tibetan (10), Tibeto-Burman (8), Himalayish (Bodic) (4)

\begin{tabular}{|l|l|l|}
\hline Chepang & Nepal & ma? \\
\hline Limbu & Nepal & oo? \\
\hline Newar(i) & Nepal & khah \\
\hline Sherpa & Nepal & jeeah \\
\hline
\end{tabular}

Sino-Tibetan (10), Tibeto-Burman (8), Jingpho-Konyak-Bodo (1)

\begin{tabular}{|l|l|l|}
\hline Chang Naga & India & háu \\
\hline
\end{tabular}

Sino-Tibetan (10), Tibeto-Burman (8), Kuki-Chin-Naga (1)

\begin{tabular}{|l|l|l|}
\hline Sumi Naga & India & ih \\
\hline
\end{tabular}

Sino-Tibetan (10), Tibeto-Burman (8), Lolo-Burmese (2)

\begin{tabular}{|l|l|l|}
\hline Akha & Myanmar, Thailand & yuh mah \\
\hline Burmese & Myanmar, Bangladesh & hou?ke, hou?pade \\
\hline
\end{tabular}

Hmong-Mien (1)

\begin{tabular}{|l|l|l|}
\hline Hmong & China, Thailand & huv \\
\hline
\end{tabular} 
Austro-Asiatic (10), Mon-Khmer (10), Aslian (5)

\begin{tabular}{|l|l|l|}
\hline Jah Hut & Malaysia & jeh \\
\hline Kensiu & Malaysia & hi?ih \\
\hline (Perak) Semai & Malaysia & éh-é \\
\hline (Ulu Kampar) Semai & Malaysia & hã \\
\hline Temiar & Malaysia & tahatna \\
\hline
\end{tabular}

Austro-Asiatic (10), Mon-Khmer (10), Eastern Mon-Khmer (4), Bahnaric (3)

\begin{tabular}{|l|l|l|}
\hline Bahnar & Viet Nam & höm öi, hám öi \\
\hline Sedang & Viet Nam & hom \\
\hline Stieng & Viet Nam & öh \\
\hline
\end{tabular}

Austro-Asiatic (10), Mon-Khmer (10), Eastern Mon-Khmer (4), Katuic (1)

\section{\begin{tabular}{|l|l|l|}
\hline Pacoh & Viet Nam & ?u \\
\hline
\end{tabular}}

Austro-Asiatic (10), Mon-Khmer (10), Northern Mon-Khmer (1), Khmuic (1)

\begin{tabular}{|l|l|l|}
\hline Khmu & Laos, Viet Nam & he \\
\hline
\end{tabular}

Austronesian (133), Malayo-Polynesian (133), Bali-Sasak (1)

\begin{tabular}{|l|l|l|}
\hline Sasak & Indonesia & ao?, au? \\
\hline
\end{tabular}

Austronesian (133), Malayo-Polynesian (133), Barito (Borneo) (3)

\begin{tabular}{|l|l|l|}
\hline Dohoi & Indonesia & ijo? \\
\hline Ma'anyan (Dayak) & Indonesia & hi?ai \\
\hline Ngaju (Dayak) & Indonesia & joh \\
\hline
\end{tabular}

Austronesian (133), Malayo-Polynesian (133), Central-Eastern (86), Central Malayo-Polynesian (18), Aru (1)

\begin{tabular}{|l|l|l|}
\hline Kola & Indonesia & 藏 \\
\hline
\end{tabular}

Austronesian (133), Malayo-Polynesian (133), Central-Eastern (86), Central Malayo-Polynesian (18), Bima-Sumba (2)

\begin{tabular}{|l|l|l|}
\hline Ende & Indonesia & o?oh \\
\hline Kambera & Indonesia & a?a \\
\hline
\end{tabular}


Austronesian (133), Malayo-Polynesian (133), Central-Eastern (86), Central Malayo-Polynesian (18), Central Maluku (11)

\begin{tabular}{|l|l|l|}
\hline Amahai & Indonesia & helo \\
\hline Ambelau & Indonesia & ehe \\
\hline Asilulu & Indonesia & ho-o \\
\hline Boano & Indonesia & ode? \\
\hline Buru & Indonesia & ehe \\
\hline Elpaputih & Indonesia & i?a \\
\hline Geser-Gorom & Indonesia & helo \\
\hline Saparua & Indonesia & ijawahi, hello \\
\hline Sapolewa Seram & Indonesia & i?jo, he? \\
\hline Sepa & Indonesia & helo \\
\hline Taliabu & Indonesia & ihi \\
\hline
\end{tabular}

Austronesian (133), Malayo-Polynesian (133), Central-Eastern (86), Central Malayo-Polynesian (18), Timor (4)

\begin{tabular}{|l|l|l|}
\hline Bilba & Indonesia & hei \\
\hline Sika & Indonesia & ehe \\
\hline Tetun & Indonesia & he?, ho(u) \\
\hline Uab Meto & Indonesia & hao, hé \\
\hline
\end{tabular}

Austronesian (133), Malayo-Polynesian (133), Central-Eastern (86), Eastern Malayo-Polynesian (68), Oceanic (68), Admiralty Islands (15)

\begin{tabular}{|c|c|c|}
\hline Bipi & Papua New Guinea & $\varepsilon$ che \\
\hline Kele & Papua New Guinea & he?é, (e)'he \\
\hline Khehek & Papua New Guinea & he? \\
\hline Koro & Papua New Guinea & ehe \\
\hline Kurti & Papua New Guinea & ehe \\
\hline Leipon & Papua New Guinea & che \\
\hline Lele & Papua New Guinea & عh\&? \\
\hline Likum & Papua New Guinea & ehe \\
\hline Loniu & Papua New Guinea & $\varepsilon$ che \\
\hline Lou & Papua New Guinea & sa? \\
\hline Mokerang & Papua New Guinea & 'che \\
\hline Mondropolon & Papua New Guinea & sa? \\
\hline Nali & Papua New Guinea & $\varepsilon$ Ph $\varepsilon$ \\
\hline Nyindrou & Papua New Guinea & عhع? \\
\hline Wuvulu-Aua & Papua New Guinea & hi?i \\
\hline
\end{tabular}


Austronesian (133), Malayo-Polynesian (133), Central-Eastern (86), Eastern Malayo-Polynesian (68), Oceanic (68), Central-Eastern Oceanic (20), Remote Oceanic (13), Central Pacific (9), East Fijian-Polynesian (8)

\begin{tabular}{|l|l|l|}
\hline Futuna-Aniwa & Vanuatu & ho \\
\hline Hawaiian & United States & Pae \\
\hline Maori & New Zealand & ?aae, ?ee \\
\hline Nukuria & Papua New Guinea & i'no? \\
\hline Rarotongan & Cook Islands & ?ae \\
\hline Rennell-Belona & Solomon Islands & ?oo \\
\hline Samoan & Samoa & ?oe, ?ii \\
\hline Tongan & Tonga & ?io \\
\hline
\end{tabular}

Austronesian (133), Malayo-Polynesian (133), Central-Eastern (86), Eastern Malayo-Polynesian (68), Oceanic (68), Central-Eastern Oceanic (20), Remote Oceanic (13), Central Pacific (9), West Fijian-Rotuman (1)

\section{\begin{tabular}{l|l|l|} 
Rotuman & Fiji & ?i, ?o \\
\hline
\end{tabular}}

Austronesian (133), Malayo-Polynesian (133), Central-Eastern (86), Eastern Malayo-Polynesian (68), Oceanic (68), Central-Eastern Oceanic (20), Remote Oceanic (13), Micronesian (2)

\begin{tabular}{|l|l|l|}
\hline Kosraean & Micronesia & ahok \\
\hline Nauruan & Nauru & eh \\
\hline
\end{tabular}

Austronesian (133), Malayo-Polynesian (133), Central-Eastern (86), Eastern Malayo-Polynesian (68), Oceanic (68), Central-Eastern Oceanic (20), Remote Oceanic (13), North and Central Vanuatu (2)

\begin{tabular}{|l|l|l|}
\hline (East) Ambae & Vanuatu & ho?o \\
\hline Sakao & Vanuatu & hao \\
\hline
\end{tabular}

Austronesian (133), Malayo-Polynesian (133), Central-Eastern (86), Eastern Malayo-Polynesian (68), Oceanic (68), Central-Eastern Oceanic (20), South Vanuatu (3)

\begin{tabular}{|l|l|l|}
\hline Aneityum & Vanuatu & ho \\
\hline Kwamera & Vanuatu & owah \\
\hline Lenakel & Vanuatu & ouaah \\
\hline
\end{tabular}

Austronesian (133), Malayo-Polynesian (133), Central-Eastern (86), Eastern Malayo-Polynesian (68), Oceanic (68), Central-Eastern Oceanic (20), Southeast Solomonic (4)

\begin{tabular}{|l|l|l|}
\hline Arosi & Solomon Islands & Pa?a, ?e?e, ?uu \\
\hline Bughotu & Solomon Islands & 'hi?i, 'he?e \\
\hline Kwaio & Solomon Islands & a?a \\
\hline Kwara'ae & Solomon Islands & ?iu \\
\hline
\end{tabular}


Austronesian (133), Malayo-Polynesian (133), Central-Eastern (86), Eastern Malayo-Polynesian (68), Oceanic (68), Western Oceanic (33), Meso Melanesian (8), New Ireland (8)

\begin{tabular}{|l|l|l|}
\hline Cheke Holo & Solomon Islands & he?e \\
\hline Halia & Papua New Guinea & geha \\
\hline Kokota & Solomon Islands & ehe \\
\hline Nehan & Papua New Guinea & 'hawun \\
\hline Petats & Papua New Guinea & oai? \\
\hline Saposa & Papua New Guinea & 'eje? \\
\hline Solos & Papua New Guinea & ?eh \\
\hline Tinputz & Papua New Guinea & kè? \\
\hline
\end{tabular}

Austronesian (133), Malayo-Polynesian (133), Central-Eastern (86), Eastern Malayo-Polynesian (68), Oceanic (68), Western Oceanic (33), North New Guinea (11), Huon Gulf (6)

\begin{tabular}{|l|l|l|}
\hline Adzera & Papua New Guinea & hai \\
\hline Bugawac & Papua New Guinea & ai? \\
\hline Kela & Papua New Guinea & $? \varepsilon ? \varepsilon$ \\
\hline Wampar & Papua New Guinea & Pijo \\
\hline Yabem & Papua New Guinea & ae? \\
\hline Zenag & Papua New Guinea & $\beta a ?$ \\
\hline
\end{tabular}

Austronesian (133), Malayo-Polynesian (133), Central-Eastern (86), Eastern Malayo-Polynesian (68), Oceanic (68), Western Oceanic (33), North New Guinea (11), Ngero-Vitiaz (5)

\begin{tabular}{|l|l|l|}
\hline Arop-Lokep & Papua New Guinea & $\varepsilon ?$ \\
\hline Bebeli & Papua New Guinea & e?e \\
\hline Gimi & Papua New Guinea & ehe \\
\hline Karnai & Papua New Guinea & bio? \\
\hline Tami & Papua New Guinea & i? \\
\hline
\end{tabular}


Austronesian (133), Malayo-Polynesian (133), Central-Eastern (86), Eastern Malayo-Polynesian (68), Oceanic (68), Western Oceanic (33), Papuan Tip (14)

\begin{tabular}{|l|l|l|}
\hline Anuki & Papua New Guinea & Peqa \\
\hline 'Auhelawa & Papua New Guinea & ehewa \\
\hline Boselewa & Papua New Guinea & i?wa \\
\hline Buhutu & Papua New Guinea & ihi \\
\hline Bunamu & Papua New Guinea & 'ehe(wa) \\
\hline Doga & Papua New Guinea & ?ona \\
\hline Duau & Papua New Guinea & ćhe \\
\hline Gumawana & Papua New Guinea & go? \\
\hline Gweda & Papua New Guinea & hímad $\Lambda$ \\
\hline Haigwai & Papua New Guinea & e?e?e \\
\hline Iduna & Papua New Guinea & ehe \\
\hline Keapara & Papua New Guinea & e?e \\
\hline Molima & Papua New Guinea & Pao \\
\hline Sewa Bay & Papua New Guinea & 'ehe \\
\hline
\end{tabular}

Austronesian (133), Malayo-Polynesian (133), Chamorro (1)

\section{\begin{tabular}{|l|l|l|}
\hline Chamorro & Guam, Northern Mariana Islands & hu?u \\
\hline
\end{tabular}}

Austronesian (133), Malayo-Polynesian (133), Kayan-Murik (2)

\begin{tabular}{|l|l|l|}
\hline Aoheng & Indonesia & ha?u \\
\hline Busang Kayan & Indonesia & io? \\
\hline
\end{tabular}

Austronesian (133), Malayo-Polynesian (133), Malayic (Sundic) (9)

\begin{tabular}{|l|l|l|}
\hline Banjar & Indonesia, Malaysia & i?ih \\
\hline Chru & Viet Nam & hèh \\
\hline Jakun & Malaysia & jeh, iah, ja? \\
\hline Jambi (Ulu) Malay & Indonesia & au? \\
\hline Jarai & Viet Nam & hoi, hom \\
\hline Pasemah & Indonesia & a?u \\
\hline Rade & Viet Nam & $\mathrm{m} \wedge \mathrm{h}$ \\
\hline Serawai & Indonesia & a?u \\
\hline Western Cham & Cambodia & hu, haij \\
\hline
\end{tabular}

Austronesian (133), Malayo-Polynesian (133), Meso Philippine (3)

\begin{tabular}{|l|l|l|}
\hline Aklanon & Philippines & huo \\
\hline Mansaka & Philippines & u?u \\
\hline Tagalog & Philippines & 'o:?o \\
\hline
\end{tabular}


Austronesian (133), Malayo-Polynesian (133), Northwest (5), North Sarawakan (3)

\begin{tabular}{|l|l|l|}
\hline Kelabit & Malaysia, Indonesia & he?-eh \\
\hline Kenyah & Indonesia & ăhà? \\
\hline Tring & Malaysia & e?a \\
\hline
\end{tabular}

Austronesian (133), Malayo-Polynesian (133), Northwest (5), Sabahan (2)

\begin{tabular}{|l|l|l|}
\hline Dusun & Malaysia & o?oh \\
\hline Kadazan & Malaysia & o?oh \\
\hline
\end{tabular}

Austronesian (133), Malayo-Polynesian (133), South Mindanao (1)

\section{\begin{tabular}{|l|l|l|}
\hline Tiruray & Philippines & ho?o \\
\hline
\end{tabular}}

Austronesian (133), Malayo-Polynesian (133), Southern Philippine (1)

\begin{tabular}{|l|l|l|}
\hline Dibabawon Manobo & Philippines & ว?ว \\
\hline
\end{tabular}

Austronesian (133), Malayo-Polynesian (133), Sulawesi (19)

\begin{tabular}{|c|c|c|}
\hline Banggai & Indonesia & ò?ò \\
\hline Coastal Konjo & Indonesia & io? \\
\hline Dampelas & Indonesia & hije \\
\hline Kulisusu & Indonesia & ũũhũ \\
\hline Laiyolo & Indonesia & ijo-uh \\
\hline Mori & Indonesia & huumbee \\
\hline Padoe & Indonesia & humbe \\
\hline (Petapa) Taje & Indonesia & ho?o \\
\hline Ratahan & Indonesia & u-hu \\
\hline Selayar & Indonesia & ijo-uh \\
\hline Suwawa & Indonesia & oo? \\
\hline (Taruna) Sangir & Indonesia & eien \\
\hline Tolaki & Indonesia & oho \\
\hline Tomini & Indonesia & Reie \\
\hline Tondano & Indonesia & uhu? \\
\hline Tontemboan & Indonesia & e?en \\
\hline Tukang Besi & Indonesia & oho \\
\hline Waru & Indonesia & hun \\
\hline Wawonii & Indonesia & hoo \\
\hline
\end{tabular}

Austronesian (133), Malayo-Polynesian (133), Sumatra (2)

\begin{tabular}{|l|l|l|}
\hline Mentawai & Indonesia & o?o \\
\hline Nias & Indonesia & ahe, ja?ia \\
\hline
\end{tabular}


West Papuan (1), North Halmahera (1)

\begin{tabular}{|l|l|l|}
\hline Galela & Indonesia & hiaa \\
\hline
\end{tabular}

Sko (2), Krisa (1)

\begin{tabular}{|l|l|l|}
\hline Warapu & Papua New Guinea & 'a?o \\
\hline
\end{tabular}

Sko (2), Vanimo (Western Sko) (1)

\begin{tabular}{|l|l|l|}
\hline Skou & Indonesia & ?æ \\
\hline
\end{tabular}

Torricelli (6), Kombio-Arapesh (3)

\begin{tabular}{|l|l|l|}
\hline Bumbita Arapesh & Papua New Guinea & o?u?c \\
\hline Wom & Papua New Guinea & auhe \\
\hline Yambes & Papua New Guinea & oho \\
\hline
\end{tabular}

Torricelli (6), Marienberg (2)

\begin{tabular}{|l|l|l|}
\hline Buna & Papua New Guinea & joo? \\
\hline Kamasau & Papua New Guinea & e?a \\
\hline
\end{tabular}

Torricelli (6), Wapei-Palei (1)

\begin{tabular}{|l|l|l|}
\hline Urat & Papua New Guinea & he \\
\hline
\end{tabular}

Kwomtari-Baibai (1)

\begin{tabular}{|l|l|l|}
\hline Baibai & Papua New Guinea & wə? \\
\hline
\end{tabular}

Left May (1)

\begin{tabular}{|l|l|l|}
\hline Iteri & Papua New Guinea & wowo? \\
\hline
\end{tabular}

Sepik-Ramu (8), Ramu (2), Ramu Proper (2)

\begin{tabular}{|l|l|l|}
\hline Arafundi & Papua New Guinea & ?o \\
\hline Kire & Papua New Guinea & aha \\
\hline
\end{tabular}

Sepik-Ramu (8), Sepik (6), Middle Sepik (2)

\begin{tabular}{|l|l|l|}
\hline Kwoma & Papua New Guinea & hehe \\
\hline Manambu & Papua New Guinea & haa-joú \\
\hline
\end{tabular}


Sepik-Ramu (8), Sepik (6), Sepik Hill (4)

\begin{tabular}{|l|l|l|}
\hline Alamblak & Papua New Guinea & ?oa \\
\hline Bisis & Papua New Guinea & ?e?ej \\
\hline Niksek & Papua New Guinea & i'pahe \\
\hline Sumariup & Papua New Guinea & ?ejo \\
\hline
\end{tabular}

Trans-New Guinea (52), Main Section (32), Central and Western (23), Angan (1)

\begin{tabular}{|l|l|l|}
\hline Baruya & Papua New Guinea & ja?jo \\
\hline
\end{tabular}

Trans-New Guinea (52), Main Section (32), Central and Western (23), Central and South New Guinea-Kutubuan (3)

\begin{tabular}{|l|l|l|}
\hline Bimin & Papua New Guinea & Pa'o \\
\hline Kasua & Papua New Guinea & 'ẽhẽ \\
\hline Konai & Papua New Guinea & he' $l æ$ \\
\hline
\end{tabular}

Trans-New Guinea (52), Main Section (32), Central and Western (23), East New Guinea Highlands (11), Central (1), Chimbu (1)

\begin{tabular}{|l|l|l|}
\hline Kuman & Papua New Guinea & o?o \\
\hline
\end{tabular}

Trans-New Guinea (52), Main Section (32), Central and Western (23), East New Guinea Highlands (11), East-Central (7)

\begin{tabular}{|l|l|l|}
\hline Alekano & Papua New Guinea & oo? \\
\hline Benabena & Papua New Guinea & ó?jo \\
\hline Gende & Papua New Guinea & o?o \\
\hline Inoke-Yate & Papua New Guinea & he \\
\hline Kanite & Papua New Guinea & he \\
\hline Keyagana & Papua New Guinea & he \\
\hline Yagaria & Papua New Guinea & he, hißa \\
\hline
\end{tabular}

Trans-New Guinea (52), Main Section (32), Central and Western (23), East New Guinea Highlands (11), West-Central (3)

\begin{tabular}{|l|l|l|}
\hline Angal & Papua New Guinea & ?æ̃ \\
\hline Angal Heneng & Papua New Guinea & $\varepsilon \tilde{h}$ \\
\hline Huli & Papua New Guinea & hee \\
\hline
\end{tabular}


Trans-New Guinea (52), Main Section (32), Central and Western (23), Huon-Finisterre (6)

\begin{tabular}{|l|l|l|}
\hline Abaga & Papua New Guinea & o?zo \\
\hline Asaro'o & Papua New Guinea & go?on \\
\hline Awara & Papua New Guinea & hi'?i \\
\hline Forak & Papua New Guinea & o? \\
\hline Kâte & Papua New Guinea & oho? \\
\hline Mape & Papua New Guinea & o'o? \\
\hline
\end{tabular}

Trans-New Guinea (52), Main Section (32), Central and Western (23), Marind (2)

\begin{tabular}{|l|l|l|}
\hline Kuni-Boazi & Papua New Guinea & e? \\
\hline Zimakani & Papua New Guinea & a?a \\
\hline
\end{tabular}

Trans-New Guinea (52), Main Section (32), Eastern (9), Central and Southeastern (9), Dagan (3)

\begin{tabular}{|l|l|l|}
\hline Kanasi & Papua New Guinea & o?a \\
\hline Mapena & Papua New Guinea & ?e \\
\hline Turaka & Papua New Guinea & ?e \\
\hline
\end{tabular}

Trans-New Guinea (52), Main Section (32), Eastern (9), Central and Southeastern (9), Goilalan (1)

\section{\begin{tabular}{|l|l|l|}
\hline Fuyug & Papua New Guinea & e?e \\
\hline
\end{tabular}}

Trans-New Guinea (52), Main Section (32), Eastern (9), Central and Southeastern (9), Koiarian (3)

\begin{tabular}{|l|l|l|}
\hline Ese & Papua New Guinea & i?a, ka?ivo \\
\hline Grass Koiari & Papua New Guinea & n?n, o?e \\
\hline Ömie & Papua New Guinea & iu?u \\
\hline
\end{tabular}

Trans-New Guinea (52), Main Section (32), Eastern (9), Central and Southeastern (9), Kwalean (1)

\begin{tabular}{|l|l|l|}
\hline Uare & Papua New Guinea & 'o? \\
\hline
\end{tabular}

Trans-New Guinea (52), Main Section (32), Eastern (9), Central and Southeastern (9), Mailuan (1)

\begin{tabular}{|l|l|l|}
\hline Mailu & Papua New Guinea & e?e \\
\hline
\end{tabular}


Trans-New Guinea (52), Eleman (4)

\begin{tabular}{|l|l|l|}
\hline Kaki Ae & Papua New Guinea & $\tilde{\varepsilon}$ h $\tilde{\varepsilon}$ \\
\hline Opao & Papua New Guinea & ehe \\
\hline Tairuma & Papua New Guinea & ahae \\
\hline Toaripi & Papua New Guinea & a?a \\
\hline
\end{tabular}

Trans-New Guinea (52), Madang-Adelbert Range (10), Adelbert Range (2)

\begin{tabular}{|l|l|l|}
\hline Moresada & Papua New Guinea & ə?ə \\
\hline Tauya & Papua New Guinea & o?o \\
\hline
\end{tabular}

Trans-New Guinea (52), Madang-Adelbert Range (10), Madang (8), Mabuso (5)

\begin{tabular}{|l|l|l|}
\hline Garus & Papua New Guinea & ?o?, æ2 \\
\hline Girawa & Papua New Guinea & hoo \\
\hline Rempi & Papua New Guinea & ac? \\
\hline Samosa & Papua New Guinea & oh \\
\hline Wamas & Papua New Guinea & ?u?u \\
\hline
\end{tabular}

Trans-New Guinea (52), Madang-Adelbert Range (10), Madang (8), Rai Coast (3)

\begin{tabular}{|l|l|l|}
\hline Ganglau & Papua New Guinea & oh \\
\hline Sam & Papua New Guinea & o? \\
\hline Yabong & Papua New Guinea & o?o \\
\hline
\end{tabular}

Trans-New Guinea (52), Northern (3), Border (3)

\begin{tabular}{|l|l|l|}
\hline Amanab & Papua New Guinea & ?ee \\
\hline Sowanda & Papua New Guinea & jəə? \\
\hline Waris & Papua New Guinea, Indonesia & ว̃?ว̃ \\
\hline
\end{tabular}

Trans-New Guinea (52), Trans-Fly-Bulaka River (3)

\begin{tabular}{|l|l|l|}
\hline Bamu & Papua New Guinea & e?e \\
\hline Northeast Kiwai & Papua New Guinea & $? \varepsilon \varepsilon$ \\
\hline Waboda & Papua New Guinea & i?o \\
\hline
\end{tabular}

East Papuan (3), Yele-Solomons-New Britain (1), New Britain (1), Kuot (1)

\begin{tabular}{|l|l|l|}
\hline Kuot & Papua New Guinea & (?)aa(?) \\
\hline
\end{tabular}

East Papuan (3), Bougainville (2), East (2)

\begin{tabular}{|l|l|l|}
\hline Naasioi & Papua New Guinea & ee? \\
\hline Sibe & Papua New Guinea & 'cu? \\
\hline
\end{tabular}


Australian (6), Pama-Nyungan (6)

\begin{tabular}{|l|l|l|}
\hline Djinang & Australia & ja?aw \\
\hline Wik-Mungkan & Australia & ee? \\
\hline Worimi & Australia (extinct) & njee-hu \\
\hline Yugambal & Australia (extinct) & geh \\
\hline
\end{tabular}

Australian (6), (Pama-Nyungan,) Kulin (2)

\begin{tabular}{|l|l|l|}
\hline Colac (Gulidjan) & Australia & aha \\
\hline Wathawurrung & Australia & aha, ha ha, eh eh \\
\hline
\end{tabular}

Eskimo-Aleut (1)

\begin{tabular}{|l|l|l|}
\hline Pacific Gulf Yupik & United States & aa?a \\
\hline
\end{tabular}

Na-Dene (5), Nuclear Na-Dene (5), Athapaskan-Eyak (5)

\begin{tabular}{|l|l|l|}
\hline Apache & United States & ha?oh, ha?ah \\
\hline Kato & United States (extinct) & hee?uu? \\
\hline Navajo & United States & aou?, aoo? \\
\hline Tanaina & United States & aa? \\
\hline Tsetsaut & Canada (extinct) & haa ah \\
\hline
\end{tabular}

Algic (10), Algonquian (9)

\begin{tabular}{|l|l|l|}
\hline Cheyenne & United States & héehe?c, haáhe \\
\hline Chippewa & United States & heh \\
\hline Cree & Canada, United States & e?he?, âha, îhî \\
\hline Malecite-Passamaquoddy & Canada, United States & aha \\
\hline Micmac & Canada, United States & 'eehe, e?e \\
\hline Montagnais & Canada & ehe \\
\hline Naskapi & Canada & niihiij \\
\hline Potawatomi & United States, Canada & e?he \\
\hline Western Abnaki & Canada, United States & ôhô( $(\hat{o})$ \\
\hline
\end{tabular}

Algic (10), Wiyot (1)

\begin{tabular}{|l|l|l|}
\hline Wiyot & United States (extinct) & hè \\
\hline
\end{tabular}

French-Cree mixed language (Indo-European, Italic, Romance + Algic, Algonquian) (1)

\begin{tabular}{|l|l|l|}
\hline Michif & United States, Canada & aenhenk \\
\hline
\end{tabular} 
Iroquoian (4), Northern Iroquoian (4)

\begin{tabular}{|c|c|c|}
\hline Cayuga & Canada, United States & éhé \\
\hline Mohawk & Canada, United States & hén \\
\hline Seneca & United States, Canada & 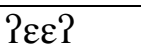 \\
\hline Tuscarora & Canada, United States & heh-heh \\
\hline
\end{tabular}

Muskogean (3)

\begin{tabular}{|l|l|l|}
\hline Alabama & United States & how \\
\hline Choctaw & United States & ãh \\
\hline Muskogee & United States & henká, ho \\
\hline
\end{tabular}

Gulf (2)

\begin{tabular}{|l|l|l|}
\hline Atakapa & United States (extinct) & ha(ha) \\
\hline Chitimacha & United States (extinct) & aha \\
\hline
\end{tabular}

Siouan (7)

\begin{tabular}{|l|l|l|}
\hline Biloxi & United States (extinct) & he \\
\hline Catawba & United States (extinct) & himba \\
\hline Dakota & United States & ha(n) \\
\hline Hidatsa & United States & hao \\
\hline Iowa-Oto & United States (extinct) & hunje \\
\hline Lakota & United States & haw, han \\
\hline Osage & United States & ho- \\
\hline
\end{tabular}

Kiowa Tanoan (2)

\begin{tabular}{|l|l|l|}
\hline Jemez & United States & hah \\
\hline Kiowa & United States & haa? \\
\hline
\end{tabular}

Uto-Aztecan (21), Northern Uto-Aztecan (10), Hopi (1)

\begin{tabular}{|l|l|l|}
\hline Hopi & United States & as?a, ta?a \\
\hline
\end{tabular}

Uto-Aztecan (21), Northern Uto-Aztecan (10), Numic (6)

\begin{tabular}{|l|l|l|}
\hline Comanche & United States & haa, hah \\
\hline Kawaiisu & United States & hu?uu \\
\hline Mono & United States & ha?, hühü \\
\hline Northern Paiute & United States & aha, ha?a \\
\hline Shoshoni & United States & hãã \\
\hline Ute-Southern Paiute & United States & hưứ, hi?i \\
\hline
\end{tabular}


Uto-Aztecan (21), Northern Uto-Aztecan (10), Takic (2)

\begin{tabular}{|l|l|l|}
\hline Cahuilla & United States & hée \\
\hline Luiseño & United States & ohoo \\
\hline
\end{tabular}

Uto-Aztecan (21), Northern Uto-Aztecan (10), Tubatulabal (1)

\begin{tabular}{|l|l|l|}
\hline Tübatulabal & United States & han \\
\hline
\end{tabular}

Uto-Aztecan (21), Southern Uto-Aztecan (11), Aztecan (2)

\begin{tabular}{|l|l|l|}
\hline Pipil & El Salvador & eehe \\
\hline Southeastern Puebla Nahuatl & Mexico & e'he $^{\prime}$ \\
\hline
\end{tabular}

Uto-Aztecan (21), Southern Uto-Aztecan (11), Sonoran (9), Cahita (4)

\begin{tabular}{|l|l|l|}
\hline Eudeve & Mexico & héve, heé, hoi éko \\
\hline Mayo & Mexico & heewi \\
\hline Opata & Mexico & haru \\
\hline Yaqui & Mexico & héewi, hehe \\
\end{tabular}

Uto-Aztecan (21), Southern Uto-Aztecan (11), Sonoran (9), Corachol (2)

\begin{tabular}{|l|l|l|}
\hline Cora & Mexico & hée \\
\hline Huichol & Mexico & húu, húu \\
\hline
\end{tabular}

Uto-Aztecan (21), Southern Uto-Aztecan (11), Sonoran (9), Tarahumaran (1)

\begin{tabular}{|l|l|l|}
\hline Tarahumara & Mexico & húri \\
\hline
\end{tabular}

Uto-Aztecan (21), Southern Uto-Aztecan (11), Sonoran (9), Tepiman (2)

\begin{tabular}{|l|l|l|}
\hline Pima Bajo & Mexico & heu?u \\
\hline Tohono O'odham & United States, Mexico & huu?u, hau?u \\
\hline
\end{tabular}

Salishan (7), Central Salish (4)

\begin{tabular}{|l|l|l|}
\hline Clallam & United States & ?aa \\
\hline Lushootseed & United States & ?i \\
\hline Southern Puget Sound Salish & United States & ?i \\
\hline Straits Salish & Canada, United States & hee?e \\
\hline
\end{tabular}

Salishan (7), Interior Salish (3)

\begin{tabular}{|l|l|l|}
\hline Coeur d'Alene & United States & hej \\
\hline Okanagan & Canada, United States & waj? \\
\hline Spokane & United States & ?a \\
\hline
\end{tabular}


Penutian (13), California Penutian (1), Wintuan (1)

\begin{tabular}{|l|l|l|}
\hline Wintu & United States & ho(o), ?ume \\
\hline
\end{tabular}

Penutian (13), Chinookan (1)

\begin{tabular}{|l|l|l|}
\hline Chinook & United States & ah-ha e-eh \\
\hline
\end{tabular}

Penutian (13), Maiduan (1)

\begin{tabular}{|l|l|l|}
\hline Maidu & United States & hee, he?u \\
\hline
\end{tabular}

Penutian (13), Plateau Penutian (2), Klamath-Modoc (1)

\begin{tabular}{|l|l|l|}
\hline Klamath-Modoc & United States & 2ii \\
\hline
\end{tabular}

Penutian (13), Plateau Penutian (2), Sahaptin (1)

\begin{tabular}{|l|l|l|}
\hline Nez Perce & United States & Pe-hé \\
\hline
\end{tabular}

Penutian (13), Yok-Utian (8), Utian (7), Costanoan (1)

\begin{tabular}{|l|l|l|}
\hline Ohlone & United States & he(ah) \\
\hline
\end{tabular}

Penutian (13), Yok-Utian (8), Utian (7), Miwokan (6)

\begin{tabular}{|l|l|l|}
\hline Amador Miwok & United States & hu \\
\hline Coast Miwok & United States & ?úu \\
\hline Mariposa Miwok & United States & huu \\
\hline Plains Miwok & United States & hûû, he-la, həəPə(h) \\
\hline Southern Sierra Miwok & United States & huuu?u \\
\hline Tuolomne Miwok & United States & hu \\
\hline
\end{tabular}

Penutian (13), Yok-Utian (8), Yokuts (1)

\begin{tabular}{|l|l|l|}
\hline Yokuts & United States & hò, hò(o)we, hò(u)hu, hûhu, hûn, hân, hòn(hu), houu \\
\hline
\end{tabular}

Hokan (9), Esselen-Yuman (5)

\begin{tabular}{|l|l|l|}
\hline Cocopa & Mexico, United States & RiiÝí, Rãã \\
\hline Esselen & United States (extinct) & i?ké \\
\hline Havasupai-Walapai-Yavapai & United States & e? \\
\hline Kiliwa & Mexico & ?haa \\
\hline Kumiai & Mexico, United States & Pe-en \\
\hline
\end{tabular}


Hokan (9), Northern (1), Karok-Shasta (1)

\begin{tabular}{|l|l|l|}
\hline Achumawi & United States & há \\
\hline
\end{tabular}

Hokan (9), Salinan-Seri (1)

\begin{tabular}{|l|l|l|}
\hline Seri & Mexico & jo'?aa \\
\hline
\end{tabular}

Hokan (9), Tequistlatecan (1)

\begin{tabular}{|l|l|l|}
\hline Chontal & Mexico & hé \\
\hline
\end{tabular}

Hokan (9), Washo (1)

\begin{tabular}{|l|l|l|}
\hline Washo & United States & je? \\
\hline
\end{tabular}

Yuki (2)

\begin{tabular}{|l|l|l|}
\hline Wappo & United States (extinct) & Yúîih \\
\hline Yuki & United States (extinct) & Rããhã?, hãwha?, ?ãh \\
\hline
\end{tabular}

Chumash (1)

\begin{tabular}{|l|l|l|}
\hline Chumash & United States (extinct) & ho, hâ?me, ?i? \\
\hline
\end{tabular}

Oto-Manguean (13), Amuzgoan (1)

\begin{tabular}{|l|l|l|}
\hline Amuzgo & Mexico & ?aha \\
\hline
\end{tabular}

Oto-Manguean (13), Mixtecan (2)

\begin{tabular}{|l|l|l|}
\hline San Miguel el Grande Mixtec(o) & Mexico & hãã \\
\hline Santa María Zacatepec Mixtec(o) & Mexico & hùu \\
\hline
\end{tabular}

Oto-Manguean (13), Otopamean (4)

\begin{tabular}{|l|l|l|}
\hline Atzingo Matlatzinca & Mexico & haa \\
\hline Mazahua & Mexico & hã(gã) \\
\hline Mezquital Otomi & Mexico & aha \\
\hline Otomi & Mexico & hã(hã) \\
\hline
\end{tabular}

Oto-Manguean (13), Popolocan (3)

\begin{tabular}{|l|l|l|}
\hline Ixcatec & Mexico & hã $^{2} \tilde{a}^{3}$ \\
\hline Mazatec(o) & Mexico & hao \\
\hline Popoloca & Mexico & haa \\
\hline
\end{tabular}


Oto-Manguean (13), Zapotecan (3)

\begin{tabular}{|l|l|l|}
\hline Mitla Zapotec(o) & Mexico & o?(n) \\
\hline Tataltepec Chatino & Mexico & hwa?ã, tso?o \\
\hline Zapotec(o) & Mexico & ja?o \\
\hline
\end{tabular}

Totonacan (2)

\begin{tabular}{|l|l|l|}
\hline Papantla Totonac(a/o) & Mexico & hé \\
\hline Xicotepec de Juárez Totonac(a/o) & Mexico & u?wee \\
\hline
\end{tabular}

Mixe-Zoque (8)

\begin{tabular}{|l|l|l|}
\hline Coatlán Mixe & Mexico & huu \\
\hline Copainalá Zoque & Mexico & hưu \\
\hline Francisco León Zoque & Mexico & hu?u \\
\hline Mixe & Mexico & hadún \\
\hline Oluta Popoluca & Mexico & hoo \\
\hline Rayón Zoque & Mexico & hu?u \\
\hline Sayula Popoluca & Mexico & hoo \\
\hline Zoque & Mexico & ha(?)a \\
\hline
\end{tabular}

Huavean (1)

\begin{tabular}{|l|l|l|}
\hline Huave & Mexico & aha(h) \\
\hline
\end{tabular}

Mayan (18), Cholan-Tzeltalan (4)

\begin{tabular}{|l|l|l|}
\hline Chol & Mexico & t $\int \mathrm{e}$ ?i \\
\hline Ch'orti' & Guatemala & huhu \\
\hline Tzeltal & Mexico & hit \\
\hline Tzotzil & Mexico & ha?, hi? \\
\hline
\end{tabular}

Mayan (18), Huastecan (1)

\begin{tabular}{|l|l|l|}
\hline Huastec(o) & Mexico & ohni? \\
\hline
\end{tabular}

Mayan (18), Kanjobalan-Chujean (3)

\begin{tabular}{|l|l|l|}
\hline Akateko (Western Q'anjob'al) & Guatemala & haa? \\
\hline Eastern Q'anjob'al & Guatemala & haa \\
\hline Tojolabal & Mexico & ha?i, oho \\
\hline
\end{tabular}


Mayan (18), Quichean-Mameam (7)

\begin{tabular}{|l|l|l|}
\hline Ixil & Guatemala & he \\
\hline K'iche' & Guatemala & he? \\
\hline Mam & Guatemala & ho \\
\hline Poqomchi' & Guatemala & ho \\
\hline Q'eqchi' & Guatemala & eh he \\
\hline Tacanec(o) & Guatemala, Mexico & oho- \\
\hline Tektiteco & Guatemala & ?o?, ?u \\
\hline
\end{tabular}

Mayan (18), Yucatecan (3)

\begin{tabular}{|l|l|l|}
\hline Itza' & Guatemala & haa \\
\hline Lacandon & Mexico & la? \\
\hline Mopán Maya & Belize, Guatemala & hah \\
\hline
\end{tabular}

Misumalpan (1)

\begin{tabular}{|l|l|l|}
\hline Sumo-Mayangna & Nicaragua, Honduras & âwih \\
\hline
\end{tabular}

Chibchan (2), Aruak (1)

\begin{tabular}{|l|l|l|}
\hline Cogui & Colombia & aha \\
\hline
\end{tabular}

Chibchan (2), Guaymi (1)

\begin{tabular}{|l|l|l|}
\hline Ngäbere & Panama & hon \\
\hline
\end{tabular}

Choco (2)

\begin{tabular}{|l|l|l|}
\hline Epena & Colombia, Ecuador & óho \\
\hline Woun Meu & Panama, Colombia & Peera \\
\hline
\end{tabular}

Barbacoan (1), Cayapa-Colorado (1)

\begin{tabular}{|l|l|l|}
\hline Chachi & Ecuador & heen \\
\hline
\end{tabular}

Guahiban (1)

\begin{tabular}{|l|l|l|}
\hline Guahibo & Colombia, Venezuela & hãhã? \\
\hline
\end{tabular} 
Tucanoan (8)

\begin{tabular}{|l|l|l|}
\hline Carapana & Colombia, Brazil & ãhã, hau \\
\hline Cubeo & Colombia, Brazil & hu \\
\hline Desano & Brazil, Colombia & ã?ã \\
\hline Koreguaje & Colombia & h̃̃h \\
\hline Secoya & Ecuador, Peru & hau, hũhu?u \\
\hline Tanimuca-Retuarã & Colombia & ã?ã \\
\hline Tatuyo & Colombia & 'h^u(?) \\
\hline Tucano & Brazil, Colombia & hai \\
\hline
\end{tabular}

Witotoan (3), Boran (1)

\begin{tabular}{|l|l|l|}
\hline Bora & Peru & héée, huúhuu \\
\hline
\end{tabular}

Witotoan (3), Witoto (2)

\begin{tabular}{|l|l|l|}
\hline Murui Huitoto & Peru & hi, huw, hee \\
\hline Ocaina & Peru & hí, huw, hãã \\
\hline
\end{tabular}

Zaparoan (1)

\begin{tabular}{|l|l|l|}
\hline Arabela & Peru & hãã \\
\hline
\end{tabular}

Peba-Yaguan (1)

\begin{tabular}{|l|l|l|}
\hline Yagua & Peru & hoo \\
\hline
\end{tabular}

Jivaroan (2)

\begin{tabular}{|l|l|l|}
\hline Achuar-Shiwiar & Peru & ha'?aj \\
\hline Aguaruna & Peru & u' $^{\prime}$ ?ũ \\
\hline
\end{tabular}

Cahuapanan (1)

\section{\begin{tabular}{|l|l|l|}
\hline Chayahuita & Peru & i?i \\
\hline
\end{tabular}}

Panoan (7)

\begin{tabular}{|c|c|c|}
\hline Amahuaca & Peru & hนันินี \\
\hline Capanahua & Peru & hứú, hóó \\
\hline Cashinahua & Peru, Brazil & haa, hũ \\
\hline Panobo & Peru & hนินในี \\
\hline Shipibo-Conibo & Peru & hน̃นในี \\
\hline Yaminahua & Peru & นี้นีั \\
\hline Yora & Peru & uhนี \\
\hline
\end{tabular}


Quechuan (2)

\begin{tabular}{|l|l|l|}
\hline Arequipa-La Unión Quechua & Peru & ô? \\
\hline Inga & Colombia & aha \\
\hline
\end{tabular}

Aymaran (2)

\begin{tabular}{|l|l|l|}
\hline Aymara & Peru & his(a) \\
\hline Jaqaru & Peru & haa \\
\hline
\end{tabular}

Harakmbet (1)

\begin{tabular}{|l|l|l|}
\hline Amarakaeri & Peru & $\tilde{\text { ẽê? }}$ \\
\hline
\end{tabular}

Maku (2)

\begin{tabular}{|l|l|l|}
\hline Hupdë & Brazil, Colombia & $\mathrm{h} \Lambda$ ? \\
\hline Yuhup & Brazil & $\mathrm{h} \Lambda$ ? \\
\hline
\end{tabular}

Arawakan (18), Maipuran (18)

\begin{tabular}{|c|c|c|}
\hline Asháninka & Peru & he \\
\hline Ashéninka & Peru & hẽẽ \\
\hline Ashéninka Pajonal & Peru & hẽẽ \\
\hline Baure & Bolivia & hah \\
\hline Caquinte & Peru & 'hẽẽhẽ \\
\hline Chamicuro & Peru & 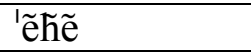 \\
\hline Ignaciano & Bolivia & he?e, (ha)?á \\
\hline Iñapari & Peru & ahamá \\
\hline Machiguenga & Peru & 'hẽẽhe, ne'?ee \\
\hline Nomatsiguenga & Peru & heé \\
\hline Parecís & Brazil & hahan \\
\hline Resígaro & Peru & háke \\
\hline Taino & Bahamas (extinct) & han(-ha?n) \\
\hline Tariano & Brazil & háw \\
\hline Wayuu & Colombia, Venezuela & ah(á) \\
\hline Yanesha' & Peru & hãã \\
\hline Yine & Peru & โนินโนี \\
\hline Yucuna & Colombia & ála \\
\hline
\end{tabular}

\section{Carib (1)}

\begin{tabular}{|l|l|l|}
\hline Wayana & Suriname & ihi, ëhë \\
\hline
\end{tabular}

Tupi (11), Arikem (1)

\begin{tabular}{|l|l|l|}
\hline Karitiâna & Brazil & h $\tilde{\Lambda} \tilde{\Lambda}$ \\
\hline
\end{tabular} 
Tupi (11), Mawe-Satere (1)

\begin{tabular}{|l|l|l|}
\hline Sateré-Mawé & Brazil & 'taa?i \\
\hline
\end{tabular}

Tupi (11), Tupi-Guarani (9)

\begin{tabular}{|c|c|c|}
\hline Avá-Canoeiro & Brazil & hiba \\
\hline Guajajára & Brazil & hê-, âê \\
\hline Guaraní & Brazil, Bolivia, Argentina & hõo, hãa, ha?e, hec \\
\hline Kamayurá & Brazil & he?en \\
\hline Tembé & Brazil & hẽ'?ẽ \\
\hline Tenharim & Brazil & ha?ã \\
\hline Urubú-Kaapor & Brazil & hã, a?é \\
\hline Wayampi & French Guiana, Brazil & оัใर̃ \\
\hline Zo’é & Brazil & $\varepsilon h \varepsilon$ \\
\hline
\end{tabular}

Macro-Ge (5), Ge-Kaingang (4)

\begin{tabular}{|l|l|l|}
\hline Kaingáng & Brazil & h \\
\hline Xavánte & Brazil & îhe \\
\hline Xerénte & Brazil & îhe, 'ehe \\
\hline Xokleng & Brazil & hõ \\
\hline
\end{tabular}

Macro-Ge (5), Maxakali (1)

\begin{tabular}{|l|l|l|}
\hline Maxakalí & Brazil & 'h̃̃?ã \\
\hline
\end{tabular}

Nambiquaran (1)

\begin{tabular}{|l|l|l|}
\hline Nambikuára & Brazil & hàjó \\
\hline
\end{tabular}

Arauan (3)

\begin{tabular}{|l|l|l|}
\hline Culina & Brazil, Peru & he?e \\
\hline Paumarí & Brazil & ha?a \\
\hline Suruahá & Brazil & hiza \\
\hline
\end{tabular}

Tacanan (4)

\begin{tabular}{|l|l|l|}
\hline Araona & Bolivia & hehe \\
\hline Cavineña & Bolivia & hehe?e \\
\hline Ese Ejja & Bolivia, Peru & e?e \\
\hline Tacana & Bolivia & hadé, ha?á, (h)e?e \\
\hline
\end{tabular}


Mataco-Guaicuru (2)

\begin{tabular}{|l|l|l|}
\hline Abipon & Argentina (extinct) & haa, hee \\
\hline Chorote & Argentina, Bolivia & xa?e \\
\hline
\end{tabular}

Isolates (5)

\begin{tabular}{|l|l|l|}
\hline Candoshi-Shapra & Peru & $(\mathrm{m}) \mathrm{a}^{\prime}$ ?aa \\
\hline Itonama & Bolivia & ãha \\
\hline Kutenai & Canada, United States & hê \\
\hline Urarina & Peru & êhẽ \\
\hline Zuni & United States & haugh \\
\hline
\end{tabular}

\section{Analysis and discussion}

The table just presented lists a total of 604 words for 'yes' taken from 512 languages belonging to 64 major linguistic families, including five isolates. In this section I give summary statistics and highlight several interesting phonological patterns evident in the data. As noted in $\$ 2$, no attempt was made to balance this sample either genetically or geographically; rather, it is a complete list of every matching form I have discovered to date. Hence, certain families are represented quite adequately, such as Austronesian with 133 languages, while others are notoriously absent. For example, there is not a single language from the Nilo-Saharan stock in my corpus. (In this paper I use the terms phylum and stock interchangeably.) This outcome is not due to any intentional purpose on my part; rather, it is a more or less accidental consequence of which parts of the world I have worked in and the concomitant collection of libraries I have had access to. In the compilation of my corpus I never avoided researching certain families or areas just because I suspected they would produce meager results. So while the sample of languages I explored is not completely random, neither is it biased in any obvious and predetermined way that would invalidate the results here.

Having clarified this point, I also now note that the relative distribution of languages in my corpus is in fact fairly well spread out among the major stocks and areas of the world. I document this in Table 2 below. From left to right I list the name of the major linguistic family, then the number of languages in that group which appear in my sample, followed by the total number of languages in that family according to Ethnologue, and finally, the corresponding percentage (number of languages from that phylum in my sample compared with total number of member languages in Ethnologue). In this table I only mention major families represented by ten or more languages in my data, and arrange them numerically from highest to lowest: 


\begin{tabular}{|l|c|c|c|}
\hline name of major stock & $\begin{array}{c}\text { number of languages } \\
\text { in my corpus }\end{array}$ & $\begin{array}{c}\text { total number of member } \\
\text { languages (Ethnologue) }\end{array}$ & percentage \\
\hline \hline Austronesian & 133 & 1246 & $10.7 \%$ \\
\hline Trans-New Guinea & 52 & 561 & 9.3 \\
\hline Indo-European & 23 & 430 & 5.3 \\
\hline Uto-Aztecan & 21 & 56 & 37.5 \\
\hline Niger-Congo & 20 & 1495 & 1.3 \\
\hline Mayan & 18 & 68 & 26.5 \\
\hline Arawakan & 18 & 49 & 36.7 \\
\hline Penutian & 13 & 23 & 56.5 \\
\hline Oto-Manguean & 13 & 172 & 7.6 \\
\hline Tupi & 11 & 60 & 18.3 \\
\hline Afro-Asiatic & 10 & 353 & 2.8 \\
\hline Sino-Tibetan & 10 & 399 & 2.5 \\
\hline Austro-Asiatic & 10 & 169 & 5.9 \\
\hline Algic & 10 & 31 & 32.3 \\
\hline \hline (overall totals) & 362 & 5112 & $7.1 \%$ \\
\hline
\end{tabular}

Table 2: Linguistic families containing at least 10 languages in my database (taken from Table 1)

In analyzing Table 2 above, it should be emphasized that the figures in column three (total number of member languages) represent the hypothetically largest possible sample sizes for those families in the world, assuming that we had available to us the corresponding data (the words for 'yes') from each language. In actual practice I was not able to exhaustively survey any of these families, so the percentages in column four correspond to preliminary hit rates (proportion of languages with a matching form) for my corpus, at an absolute minimum, i.e., assuming the complete sample sizes in column three. I am not able to supply the real hit rates per family for my study, unfortunately, since I did not keep close track of the genetic affiliations of the languages I surveyed which did not exhibit matching words for 'yes' (forms with a glottal consonant). All that I tabulated was the approximate number of misses, which added up to about 860 languages. Consequently, the complete sample size for the planet as a whole (in this paper) is roughly 1372 languages surveyed, of which the total number displayed in Table 1 (512) equals an overall matching rate of about $37.3 \%$. The quantity of languages for which I was able to ascertain the word for 'yes' (1372) corresponds to a 19.8\% sample of all the living languages in the world (6912), according to Ethnologue. This is a fairly robust figure given the magnitude of the task.

Returning now to Table 2, if my data on all the languages in the world were exhaustive, the final percentages (hit rates) in column four would all potentially increase, although to what degree is hard to know for sure. As it stands, the highest actually attested proportion (among families with ten representatives or more) is $56.5 \%$ for the Penutian stock (13 matching languages out of 23 extant). This is encouraging. On the other hand, the family with the lowest hit rate in Table 2 is Niger-Congo (1.3\%). This is symptomatic of the relatively low level of access I have had to data on African languages in general (so far). At the same time, it is not surprising that the two most numerous families in my corpus - Austronesian and Trans-New Guinea with 185 combined languages - are located in the part of the world where there is greatest linguistic diversity and density (the South Pacific). The overall number of first-order 
families exemplified by at least one language in my corpus is 64 , which amounts to $68.1 \%$ of the 94 total posited by Ethnologue. This too is a promising indicator.

I now move on to discuss a few aspects of the phonological content of the 604 words in my corpus in Table 1. The total number of glottal consonants in all forms combined is 761 , so on average each word contains about 1.3 laryngeals. Of these, 474 or $62.3 \%$ consist of [h], while the remaining $287(37.7 \%)$ are [?]. The ratio of [h] to [?] then is roughly $3: 2$. Among all these occurrences, [h] appears word-initially in 290 forms $(61.2 \%)$; the remaining 184 tokens of [h] $(38.8 \%)$ are non-initial. So [h] prefers initial over non-initial position by a margin of almost 2-to1. Indeed, nearly one-half of all the words for 'yes' in my database begin with [h]. As far as [?] is concerned, only 64 of its tokens are word-initial (22.3\%), while the remaining 223 occurrences (77.7\%) are non-initial. So [?] prefers non-initial position over initial by a margin of almost 4-to1. This is probably related to the fact that phonemic / $/$ 's in general tend not to occur wordinitially in many languages anyway.

At this point we might entertain the question, with what degree of statistical confidence can we now posit that these tendencies are significantly greater than chance? Although this issue is an important one, I am not in a position to answer it conclusively here, for two main reasons: (1) the list of data in Table 1 does not equally cover all linguistic families and geographic locations, and (2) even if my sample were ideally balanced, any global inferential test would be undermined by the fact that we don't know the actual hit-or-miss rates for each phylum of languages. In retrospect this was an unfortunate methodological oversight on my part. In a perfect world, where we had exhaustive data on every language and could thus calculate the proportion of matching forms for any subset of languages, we would be able to proceed by comparing cognate words for 'yes' within each lowest-level genetic grouping, reconstruct the corresponding proto-form and its rate of retention in each daughter language, and then work our way backwards and up each higher-order branch of the tree until we could make a definitive generalization about each stock of related languages. Obviously this is not possible in the present case, so absolute statistical probabilities, as in works such as Ringe (1995), will have to wait for future research. As it stands, the chances of getting $x$ number of look-alike hits in a large sample like this increases greatly when the corpus contains many related languages, as mine does. On the other hand, since many of the non-matching languages that I surveyed were also related to each other, this would tend to pull down the hit rates. Nevertheless, we cannot assume that these two opposing factors cancel each other out in any meaningful way, even if we could calculate them exactly. So the percentage figures I give above for the relative frequencies of [h] and [?] should only be considered very rough estimates of the corresponding population rates (for all the languages in the world). This is especially true since an expression that sounds like $u h-u h$, for a concept that means something like 'yes,' is highly susceptible to being borrowed from neighboring ethnic groups by diffusion, even if the languages are not related. What is more, in any cross-linguistic comparison of this type, a certain percentage of apparent cognates will always occur by chance no matter what (Ringe 1995). Nevertheless, having noted these caveats, we can still at the very least make a few tentative predictions or claims about what we should reasonably expect to find among the remaining languages of the world:

(1) Hypothesis 1: All else being equal, if the word for 'yes' in a particular language contains a laryngeal consonant, this is more likely to be [h] than [?]. 
Hypothesis 2: All else being equal, if the word for 'yes' in a particular language contains an $[\mathrm{h}]$, this is more likely to be word-initial than non-initial.

Hypothesis 3: All else being equal, if the word for 'yes' in a particular language contains a [?], this is more likely to be non-initial than initial.

At this point I note that the three predictions in (1) above may not necessarily be specific to the word for 'yes,' but rather may derive from more general patterns among the lexicons of the world's languages. For instance, the tendency of [?] to avoid word-initial position across the board was already mentioned (cf. hypothesis 3 ). With respect to the preference for [h] to occur morpheme-initially (cf. hypothesis 2 ), this is actually enforced as a grammatical constraint on the occurrence of [h] in most lexical items in many languages: English (Davis 1999), Cuzco Quechua (Parker and Weber 1996), Panobo or Huariapano (Parker 1994), etc. Finally, let us consider hypothesis 1, whereby [h] is preferred over [?] by a proportion of about $3: 2$ in this sample. This fact may simply be a reflection of the universal tendency of $/ \mathrm{h} /$ to appear more often than $/ 2 /$ does in phonemic inventories cross-linguistically. For example, in the UPSID database of 451 languages (Maddieson and Precoda 1992), /h/ occurs 279 times (61.9\%) and / / / 216 times (47.9\%). Similarly, in the P-base sample of 549 languages (Mielke 2006), /h/ appears in 361 inventories (65.8\%) and /2/ in only 195 (35.5\%). While these latter two samples are not as ideally balanced as $W A L S$ is, their convergence nevertheless allows us to reasonably posit that $/ \mathrm{h} /$ is probably more frequent as a phoneme in the world's languages than $/ 2 /$ is. In a sense, then, the three hypotheses in (1) are completely natural and expected.

In order to go a step further and precisely quantify these three tendencies (from (1) above), technically speaking we would really need to know the phonemic inventory of every language studied, as well as the relative frequencies of each segment in each language-specific lexicon. This monumental task is beyond the scope of this study, and is not necessary for our purposes here. Nevertheless, keeping in mind the disclaimers above about the unbalanced nature of my sample, we still have enough data to arrive at some concrete conclusions for a few of the major families from Table 2. For each stock represented by ten or more languages in my database, I counted up the total number of [h]'s and [?]'s among all their matching forms, ignoring the position of these sounds in the words where they occur. I then calculated (by phylum) the probability that the preference for one segment or the other is significantly greater than chance, using the binomial cumulative distribution (two-tailed). A similar result could also be obtained with a chi-squared test. Both of these procedures tend to be unreliable with samples consisting of less than ten tokens. In Table 3 below I display the results for those families which yielded significant results. To control for the effect of multiple comparisons (type 1 errors), I use a Bonferroni adjustment and test each contrast at an $\alpha$ level of .0036, which was arrived at by dividing .05 by 14 (the number of families listed in Table 2). Given this criterion, only five genetic groups have a preference for [h] or [?] extreme enough — and with enough tokens - to be reliable. In the following table I arrange these families by $p$ value, from lowest to highest: 


\begin{tabular}{|l|r|r|l|}
\hline family & $h$ & $?$ & $p$ \\
\hline Indo-European & 27 & 0 & .0000 \\
\hline Penutian & 28 & 7 & .0005 \\
\hline Arawakan & 24 & 5 & .0005 \\
\hline Uto-Aztecan & 32 & 10 & .0009 \\
\hline Trans-New Guinea & 17 & 43 & .0011 \\
\hline
\end{tabular}

Table 3: Language families in Table 2 which have a significant preference for one glottal consonant over the other one

As indicated in Table 3, the Indo-European languages overwhelmingly prefer to express their word for 'yes' with [h]. Every single Indo-European example in my sample contains exactly one [h] and no [?]'s. Undoubtedly this is related to the fact that few languages in this family have the phoneme / $/ 2$ at all. The only major stock which has a significant overall preference for [?] over $[\mathrm{h}]$ is Trans-New Guinea. In addition to these generalizations, there are a few other trends we can note for some of the smaller families, even though they are not statistically significant. The three Altaic words all begin with [h] and the three East Papuan words end with [?]. All eight Siouan words begin with [h] and lack [?]'s completely. The four Yuki words each contain both laryngeal consonants. The eight Mixe-Zoque forms all begin with [h], as do the eight Witotoan words. Every Panoan language has a form containing the syllable [hu]. Every Macro-Ge and Arauan word contains an $[\mathrm{h}]$.

In addition to the tendency for the word meaning 'yes' to contain one or more glottal consonants, there is another indication that these forms are somewhat special cross-linguistically in another way as well: in many cases the [h] or [?] is exceptional in that its occurrence is prohibited in the language as a whole, or at least highly restricted. I document some of these anomalies below (following the order of Table 1):

\begin{tabular}{|c|c|c|c|}
\hline language & family & 'yes' & constraint \\
\hline (East) Ambae & Austronesian & ho?o & only word with [?] \\
\hline Lenakel & Austronesian & ouaah & only word with final [h] \\
\hline Arop-Lokep & Austronesian & $\varepsilon ?$ & only three other words with [?] \\
\hline Skou & Sko & ?æ & only word with [?] \\
\hline Awara & Trans-New Guinea & hi'Pi & only word with an intervocalic [?] \\
\hline Grass Koiari & Trans-New Guinea & n?n, o?e & only words with [?] \\
\hline Kuot & East Papuan & (?)aa(?) & only word with [?] \\
\hline Djinang & Australian & ja?aw & only word with [?] \\
\hline Micmac & Algic & 'eehe & only two other words with [h] \\
\hline Montagnais & Algic & ehe & only three other words with [h] \\
\hline Achuar-Shiwiar & Jivaroan & ha'?aj & only word with an intervocalic [?] \\
\hline Panobo & Panoan & 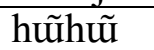 & only word with an intervocalic [h] \\
\hline Chamicuro & Arawakan & อ̃ $\tilde{\mathrm{e}}$ & only word with an intervocalic [h] \\
\hline Yanesha' & Arawakan & hãã & only word with [h] \\
\hline Candoshi-Shapra & Isolate & $(\mathrm{m}) \mathrm{a}^{\prime} \mathrm{Raa}$ & only word with an intervocalic [?] \\
\hline
\end{tabular}

Table 4: Languages having special restrictions on laryngeal consonants in general

Another case analogous to the examples in Table 4 above is provided by the English expression $u h$-uh. This is one of the few forms in the language in which the phoneme $/ \mathrm{h} /$ occurs in the middle of a morpheme; usually $/ \mathrm{h} /$ is restricted to morpheme-initial position. One other unusual detail about this word, for English, is that it is normally pronounced with nasalized vowels, even though these are not adjacent to a true nasal consonant like $/ \mathrm{m} / \mathrm{or} / \mathrm{n} /$. This is a 
classic illustration of the phenomenon of rhinoglottophilia, which Matisoff $(1975: 265)$ defines as "an affinity between the feature of nasality and the articulatory involvement of the glottis" (cf. Parker 1996, 2006). (In general this seems to be more frequent with $/ \mathrm{h} /$ than with $/ \mathrm{P} /$.) This type of irregular nasalization is also common in my database in Table 1, where 64 words $(10.6 \%$ of the total) have at least one nasalized vowel. What I do not know is whether this amount is significantly higher than the rate of occurrence of nasalized vowels overall in these languages, or for that matter in the whole world (in words other than 'yes'). Nevertheless, several of my sources for this study point out that the word for 'yes' in particular languages exceptionally contains the only contrastive or unpredictably nasalized vowel(s) in the entire lexicon. In the following table I list those cases which I have noted to date:

\begin{tabular}{|c|c|c|}
\hline language & family & 'yes' \\
\hline Kambaata & Afro-Asiatic & ใãã \\
\hline Azerbaijani & Altaic & hथ̃ \\
\hline Kola & Austronesian & ${ }^{\tilde{1} \pi \mathrm{1}}$ \\
\hline Shoshoni & Uto-Aztecan & hãã \\
\hline Ashéninka & Arawakan & hẽẽ \\
\hline Ashéninka Pajonal & Arawakan & hẽẽ \\
\hline Chamicuro & Arawakan & 'ẽโ̃̃ \\
\hline Yanesha' & Arawakan & hãã \\
\hline
\end{tabular}

Table 5: Languages in which nasalized vowels are restricted to the word for 'yes'

Before closing this discussion I have a few comments to make about vowel quality in general (not just oral vs. nasal). While this paper has focused primarily on consonants, there are also several vowel patterns which form nice generalizations. For the five universally unmarked cardinal vowels, I counted up the number of words in my corpus in which each one is the first nuclear segment. I present the results in the table below, in which I also indicate the corresponding percentage of the total of 604 words:

\begin{tabular}{|c|c|c|}
\hline segment & $\begin{array}{c}\text { number of forms as } \\
\text { first vocalic mora }\end{array}$ & $\begin{array}{c}\text { percentage of } \\
\text { total words }\end{array}$ \\
\hline \hline $\mathrm{a}$ & 188 & $31.1 \%$ \\
\hline $\mathrm{e}$ & 149 & 24.7 \\
\hline $\mathrm{o}$ & 96 & 15.9 \\
\hline $\mathrm{i}$ & 63 & 10.4 \\
\hline $\mathrm{u}$ & 29 & 4.8 \\
\hline \hline totals & 525 & 86.9 \\
\hline
\end{tabular}

Table 6: Relative frequencies of the five cardinal vowels in the corpus in Table 1

As Table 6 shows, unrounded vowels tend to be more preferred than rounded ones, which is phonologically natural - lip rounding entails an additional articulatory gesture (de Lacy 2002). Also, within each of these two sets, lower (more sonorous) vowels are more frequent than higher ones. These two tendencies joined together converge on a significant (non-random) preference for the vowel $/ \mathrm{a} /$ in the word for 'yes' cross-linguistically $\left(\chi_{(4)}^{2}=156.6, p<.0000\right)$. This is hardly surprising since /a/ is universally unmarked anyway (de Lacy 2002, 2004). Furthermore, pharyngeal and glottal consonants tend to induce lowering on adjacent vowels in general, a wellknown type of allophonic or morphophonemic conditioning via spreading (Kenstowicz 1994, McCarthy 1994). 
The last item of business is simply to list some of the most common forms in my corpus. The following table displays the eight most frequent variants of the word for 'yes' in my data, ignoring minor (secondary) details such as vowel nasalization, stress, and tone. They are ordered by decreasing number of occurrences in my database, and are exhaustive in the sense that I have not tried to balance this table by limiting the tokens to only one exemplar per family:

\begin{tabular}{|c|c|}
\hline form & $\begin{array}{c}\text { number of } \\
\text { occurrences }\end{array}$ \\
\hline \hline ehe & 26 \\
\hline haa & 25 \\
\hline he & 20 \\
\hline ha & 15 \\
\hline aha & 13 \\
\hline hee & 10 \\
\hline e?e & 10 \\
\hline a?a & 7 \\
\hline
\end{tabular}

Table 7: Relative frequencies of the most common patterns for the word 'yes' in Table 1

The canonical forms in the table above nicely summarize and illustrate the general themes I have described throughout this section.

\section{Conclusion}

In any scientific endeavor, the most important question we can ask ourselves is, why should the world be the way it is? In this case, why should there be a universal tendency for the word meaning 'yes' to contain one or more glottal consonants? One factor which undoubtedly helps to explain this phenomenon is the fact that the laryngeal place of articulation node is inherently unmarked (Lombardi 2001, 2002), based on its typical phonological behavior as placeless (Halle 1995, Ladefoged 1997, Parker 2001). In summary, Yes! there is something interesting going on here cross-linguistically, and it clearly appears to exceed random chance. That is, we have probably discovered a worldwide articulatory pattern that maps meaning onto sound in a nonarbitrary way in many languages.

\section{Acknowledgements}

This paper has received very helpful input and suggestions from many people in many places at many times. In particular, though, I would like to thank two anonymous reviewers, as well as audiences at the University of Oregon, the University of Technology in Lae (Papua New Guinea), the University of North Dakota, and the Universidad Ricardo Palma in Lima, Peru.

\section{References}

Davis, Stuart. 1999. The parallel distribution of aspirated stops and /h/ in American English. Indiana University working papers in linguistics 1.1-10.

de Lacy, Paul. 2002. The formal expression of markedness. Ph.D. dissertation. University of Massachusetts Amherst. 
de Lacy, Paul. 2004. Markedness conflation in Optimality Theory. Phonology 21/2.145-99.

Frawley, William J. (ed.) 2003. International encyclopedia of linguistics (second edition). Oxford: Oxford University Press.

Gordon, Raymond G., Jr. (ed.) 2005. Ethnologue: languages of the world (fifteenth edition). Dallas: SIL International.

Halle, Morris. 1995. Feature geometry and feature spreading. Linguistic Inquiry 26/1.1-46.

Haspelmath, Martin, Matthew S. Dryer, David Gil, and Bernard Comrie (eds.), with the collaboration of Hans-Jörg Bibiko, Hagen Jung, and Claudia Schmidt. 2005. The world atlas of language structures. Oxford: Oxford University Press.

Kenstowicz, Michael. 1994. Phonology in generative grammar. (Blackwell Textbooks in Linguistics.) Cambridge, Massachusetts and Oxford, UK: Blackwell.

Ladefoged, Peter. 1997. Linguistic phonetic descriptions. The handbook of phonetic sciences, ed. by William J. Hardcastle, and John Laver. Oxford, UK and Cambridge, Massachusetts: Blackwell. pp. 589-618.

Lombardi, Linda. 2001. Why place and voice are different: constraint-specific alternations in optimality theory. Segmental phonology in optimality theory: constraints and representations, ed. by Linda Lombardi. Cambridge: Cambridge University Press. pp. 1345.

Lombardi, Linda. 2002. Coronal epenthesis and markedness. Phonology 19/2.219-51.

Maddieson, Ian, and Kristin Precoda. 1992. UPSID. Los Angeles: UCLA phonetics laboratory.

Matisoff, James A. 1975. Rhinoglottophilia: the mysterious connection between nasality and glottality. Nasálfest (papers from a symposium on nasals and nasalization), ed. by Charles A. Ferguson, Larry M. Hyman, and John J. Ohala. Stanford: Language Universals Project, Department of Linguistics, Stanford University. pp. 265-87.

McCarthy, John J. 1994. The phonetics and phonology of Semitic pharyngeals. Phonological structure and phonetic form: papers in laboratory phonology III, ed. by Patricia A. Keating. Cambridge: Cambridge University Press. pp. 191-233.

Mielke, Jeff. 2006. P-base. http://www.u.arizona.edu/ mielke/research/pbase.html

Parker, Steve. 1994. Coda epenthesis in Huariapano. International Journal of American Linguistics 60/2.95-119.

Parker, Steve. 1996. Toward a universal form for 'yes': or, rhinoglottophilia and the affirmation grunt. Journal of Linguistic Anthropology 6/1.85-95.

Parker, Steve. 2001. Non-optimal onsets in Chamicuro: an inventory maximised in coda position. Phonology 18/3.361-86.

Parker, Steve. 2006. La rinoglotofilia y el gruñido de afirmación - una tendencia universal. Lengua y sociedad 8/1.27-56.

Parker, Steve, and David Weber. 1996. Glottalized and aspirated stops in Cuzco Quechua. International Journal of American Linguistics 62/1.70-85.

Ringe, Donald A., Jr. 1995. 'Nostratic' and the factor of chance. Diachronica 12/1.55-74.

Runner, Jennifer. 2003. "Yes" in over 550 languages. http://www.elite.net/ runner/jennifers/yes.htm

Ruhlen, Merritt. 1987. A guide to the world's languages, volume 1: classification. Stanford: Stanford University Press.

Whaley, Lindsay J. 1997. Introduction to typology: the unity and diversity of language. Thousand Oaks, California: Sage Publications. 